\title{
BMJ Open Quality Electronic prescribing: introducing a stat optional dose to improve time to antibiotic on the acute medical unit
}

\author{
Thomas Robert Edward Jones, Kim Nurse
}

To cite: Jones TRE, Nurse $\mathrm{K}$. Electronic prescribing: introducing a stat optional dose to improve time to antibiotic on the acute medical unit. BMJ Open Quality 2018;7:e000292. doi:10.1136/ bmjoq-2017-000292

KN contributed equally.

Received 16 December 2017 Accepted 17 November 2018

Check for updates

(C) Author(s) (or their employer(s)) 2018. Re-use permitted under CC BY-NC. No commercial re-use. See rights and permissions. Published by BMJ.

Acute Medical Unit, County Durham and Darlington Foundation Trust, England, UK

Correspondence to

Dr Kim Nurse;

kim.nurse@nhs.net

\section{ABSTRACT}

Darlington Memorial Hospital is a district general hospital in the North East of England. The acute medical unit (AMU) takes referrals from the emergency department and also direct from general practitioners (GPs) in the region. Research shows that early recognition and management of sepsis is key to improving outcomes and significantly decreases mortality. Having observed several cases of delayed sepsis management in patients referred from their GP, we aimed to improve time to antibiotic therapy in patients identified as having sepsis as per the National Institute for Health and Care Excellence 2016 NG51 sepsis guidelines. We analysed the time from admission to antibiotic administrations for patients referred to AMU via their GP with suspected sepsis. We found there was a significant delay between antibiotic prescribing and administration. Baseline data showed that only $36 \%$ of patients received a stat dose of antibiotic on admission. Results showed that those patients with a stat dose prescribed had a median time of $36 \mathrm{~min}$ from prescription to antibiotic compared with $98 \mathrm{~min}$ for those without. We introduced a stat antibiotic option with all antibiotic prescriptions on the electronic prescribing system, encouraging prescribers to administer a stat dose. Through focusing on antibiotic prescribing systems and practices over two PDSA cycles, we were able to improve the time from prescription to administration of antibiotics for patients with sepsis. We reduced the delay in antibiotic administration in patients with sepsis from 49 to $34 \mathrm{~min}$, an improvement of $31 \%(15 / 49)$. The percentage of patients receiving a stat dose of antibiotics increased from $36 \%$ to $63 \%$. While we acknowledge that there remains room for improvement with regards to antibiotic prescribing and sepsis management, we have made sustainable interventions with important improvements. E-prescribing systems must be evaluated and modified accordingly on a regular basis to ensure that they positively contribute to quality patient care and clinical practice.

\section{PROBLEM}

Darlington Memorial Hospital (DMH) is a district general hospital, located in the North East of England, and is part of County Durham and Darlington Foundation Trust (CDDFT). CDDFT is one of the largest integrated care providers in the country covering a patient population of approximately 650000 people. As with many acute medical units (AMUs),
$\mathrm{DMH}$ accepts patients direct from general practitioners (GPs) in the region and from the emergency department.

Having anecdotally observed several cases of delayed sepsis management in patients referred from general practice, we aimed to identify the reasons for the delay in antibiotic therapy. Timely antibiotic administration in the management of sepsis is key to reducing deaths from sepsis. While the introduction of electronic prescribing systems have numerous benefits, we noted that in some cases it may contribute to a delay in antibiotic administration due to default timings within the electronic prescribing system. ${ }^{1}$

Following our initial data collection, we aimed to improve the time from antibiotic prescription to administration by increasing the percentage of patients receiving a stat dose of antibiotic to $75 \%$ over the course of a year (by August 2017).

\section{BACKGROUND}

Each year in the UK, there are approximately 150000 admissions to hospital and 44000 deaths from sepsis. ${ }^{2}$ The mortality rate is between $28 \%$ and $50 \%$, and approximately three quarters of patients will survive sepsis if recognised and treated promptly. ${ }^{3}$ The long-term consequences must also not be ignored and even patients who survive may have a significant excess risk of mortality for a prolonged period of time. ${ }^{4}$

Historically, patients with sepsis have been identified using the systemic inflammatory response syndrome (SIRS) criteria. However, this has more recently fallen out of favour. The third international consensus definitions for sepsis and septic shock (Sepsis-3) moved away from the SIRS criteria to focus on the use of the quick sepsis-related/sequential organ failure assessment score as a risk stratification tool to assist with the identification of sepsis. Sepsis-3 set out to establish less ambiguous terminology now only using infection, sepsis and septic shock. ${ }^{5}$ 
Research shows that early recognition and management of sepsis is key to improving outcomes and significantly decreases mortality. ${ }^{2}$ Guidance states that broad-spectrum antibiotics should be given before the pathogen is identified and preferably within the first hour. ${ }^{2}$ Delivery of antibiotics within the first hour is of critical importance in maximising chances of survival. A large-scale systematic review found that giving antibiotics within 1 hour reduced mortality by $33 \%$ compared with giving antibiotics later. ${ }^{6}$ Despite widespread publicity surrounding sepsis and education within hospitals, the management of sepsis and timely antibiotics administration remains poor. ${ }^{3}$

Electronic prescribing can improve or impair clinical processes and evaluating them is key to ensure the systems are of benefit and safe. ${ }^{7}$ As part of improving sepsis management within AMU, we focused on improving timely antibiotic administration. The overall aim of the study is to evaluate whether our prescribing system has unintended consequences contributing to delays in antibiotic administration, with a view to improving this by simple modifications, ultimately improving time to antibiotic, overall sepsis management and patient mortality.

\section{BASELINE MEASUREMENT}

We initially audited the management of sepsis in patients admitted directly to AMU against the National Institute for Health and Care Excellence (NICE) 2016 sepsis guidelines. ${ }^{8}$ We did this by reviewing all the notes of patients referred direct to AMU from their GP with possible infection or sepsis in August and September 2016 and mapped their pathway from referral to administration of their first antibiotic. Using the information available on presentation to AMU (details in their GP referral and their initial observations), their lactate and creatinine (to identify acute kidney injury), we risk stratified them according to NICE guidelines on sepsis. ${ }^{8}$ We recorded their time of referral from the GP, admission time, time of first observations, and the time their lactate and creatinine results became available. We then compared these values to NICE guidelines. We calculated the time of GP referral to admission, time to first set of observations, time to lactate/creatinine results and time to antibiotics.

There were 228 patients admitted directly from their GP to AMU in August and September 2016. We identified 35 patients who received antibiotics and had at least two moderate risk factors $(15 \%)$.

Having completed this audit, we re-analysed the data looking at antibiotic prescribing practices. We found that only $36 \%(10 / 35)$ patients had a stat dose of antibiotic prescribed. The median time from antibiotic prescription to administration for those prescribed a stat dose was 35 min compared with $98 \mathrm{~min}$ for those not prescribed a stat dose.

The overall time from GP referral to antibiotic administration was $326 \mathrm{~min}$.

\section{DESIGN}

We planned to implement a change to our e-prescribing system to facilitate prescribing stat doses of antibiotics. Our e-prescribing system required two entirely separate prescriptions for stat doses and regular dosing. This required 27 clicks of a mouse to prescribe the two prescription sets. By combining the two prescriptions with an option to have an initial stat dose when prescribing the regular dosing, we reduced the number of clicks required to 19. This also provided a prompt to encourage people to give a stat dose. By simplifying the process, we hoped people would be more likely to prescribe that early initial antibiotic dose and that the presence of a prompt would make people less likely to forget to do it.

We felt introducing a better system for stat dose antibiotic prescription and administration could lead to a reduction in time to antibiotics. We wanted to have a system that prompted doctors to prescribe a stat dose, as well as a system that made it easy and encouraged them to do so. Through liaison with the lead pharmacist for e-prescribing and the lead pharmacist for antibiotic prescribing, we implemented a new simplified process with stat dose options on all antibiotic prescriptions.

We presented our results as well as an update on sepsis with a focus on Sepsis-3 and NICE guidelines to our weekly medical teaching forum. We also informed the medical staff of the planned intervention as above.

These initial interventions were fairly substantial and required longer than we expected to implement, and as such, we had to delay our reassessment by several months. Once they had been implemented and reassessed, we again met with consultants and senior nursing staff to discuss the findings and further interventions.

After our initial intervention, we observed an increase in the number of stat antibiotic doses being prescribed; however, there were still delays between these being prescribed and administered. We attributed this, in part, to poor communication between medical and nursing staff, and nursing staff being too busy with other tasks. As such, our subsequent intervention focused on education around communication, human factors and task prioritisation. We reminded medical staff of the need to inform nursing staff of any urgent tasks, and re-iterated the importance of stat antibiotic administration to nursing staff and the need to prioritise this task. However, we acknowledge that this is a weak cohort-specific intervention with limited sustainability. In order to maintain sustainability we, with the support of senior medical staff on AMU, intend to make this an ongoing part of the induction process.

\section{STRATEGY}

Following the results of our initial audit, our initial aim was to pick one specific area to improve on which could have a significant impact on the time to antibiotic administration from admission. Our data showed that very few patients received a stat dose of antibiotic on admission 
$(36 \%)$, meaning that some patients waited a number of hours post prescription to administration. This resulted in delays from sepsis diagnosis to antibiotic administration.

Our aim was to improve the time from antibiotic prescription to administration by increasing the number of patients receiving a stat dose of antibiotic to $75 \%$ over the course of a year (by August 2017).

\section{PDSA cycle 1}

Following our initial audit, we presented our results at the medical grand round to all junior doctors and medical consultants. In addition, we met with the senior nursing team on AMU to highlight the results and the need for improvement. We discussed the need for timely antibiotics and the areas in which improvement was most needed, reducing the time from admission to antibiotic and particularly from prescribing to administration.

Our aim for the first cycle was to improve rates of stat antibiotics administration through improved prescribing. This is clearly an important barrier to early antibiotic administration and one we felt we could improve. We then set about achieving this by implementing our most significant intervention. We worked with the pharmacy team to implement a stat antibiotic option on the e-prescribing system for all antibiotics, in addition to the regular antibiotic times. This system change would make it easier for doctors to prescribe stat antibiotics and in addition it would also act as a visual prompt.

Our second data point was delayed due to the time scale of implementing a stat antibiotic option on the e-prescribing system. We collected the data following this intervention and found that the number of patients with suspected sepsis receiving a stat dose of antibiotic had increased to $50 \%$.

\section{PDSA cycle 2}

Despite now having a stat antibiotic option on the e-prescribing system, acting as both a visual prompt and ensuring this action is simple, a significant number of patients still did not have a stat dose of antibiotic prescribed.

We presented the data to the nursing and medical team at an education session on the AMU, and at the medical grand round to all junior doctors and medical consultants. In addition, we discussed several other issues surrounding antibiotic prescribing and administration, including the need to prescribe a stat dose of antibiotic, further highlighting the presence of a stat antibiotic dose option and the need to inform nurses of this prescription to ensure timely antibiotic administration.

Our third data point was conducted in June, 2 months after the second. Reassuringly, the results showed an increase in the number of stat antibiotics administered $(62.5 \%)$ and an overall decrease in the time from antibiotic prescription to administration. We noted that our intervention and implemented changes have had a sustained impact on antibiotic prescribing practice, with an improvement in results at every cycle (see figure 1 ).

There are clearly still improvements to be made. We considered one possible reason for patients not having a stat dose of antibiotic prescribed was that they were due their first regular dose and therefore a stat dose is not required; we intended to further analyse these data at the next cycle. Due to unexpected delays implementing our initial intervention, we were unable to do additional PDSA cycles as we had hoped.

Displaying the data and our improvement cycles in the unit and visible to all staff has also helped to improve awareness and sustainability of the changes.

\section{RESULTS}

Our baseline measurement in August and September showed that $36 \%(10 / 35)$ patients with sepsis had a stat dose of antibiotic prescribed; that $36 \%$ had a median time from antibiotic prescription to administration of 35 min compared with $98 \mathrm{~min}$ in those without a stat dose

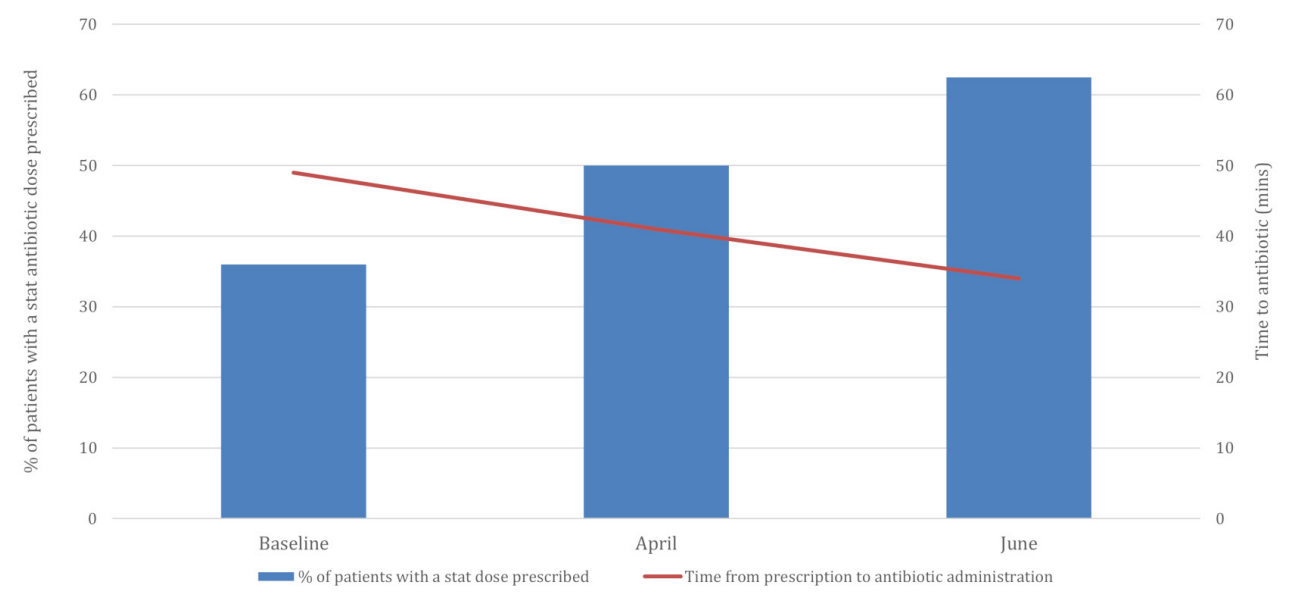

Figure 1 Number of stat doses prescribed and median time to antibiotic administration from prescription. 
prescribed. The overall median time from prescription to administration of antibiotics was $49 \mathrm{~min}$.

In April, after we had implemented our initial intervention of changing the e-prescribing system to make stat doses of antibiotics easier to prescribe, $50 \%(5 / 10)$ patients with sepsis had a stat dose prescribed. The median time for administration of antibiotics within those that received a stat dose prescription was $24 \mathrm{~min}$ compared with $50 \mathrm{~min}$ for those without. The overall median time from prescription to administration of antibiotics was $41 \mathrm{~min}$, an improvement of $16 \%(8 / 49)$ from baseline.

In June, after our second intervention of education with a focus on communication between medical and nursing teams, $63 \%(5 / 8)$ patients with sepsis had a stat dose of antibiotics prescribed. Those with a stat dose prescribed had a median time to antibiotics of $14 \mathrm{~min}$, and those without a stat prescription had a median delay of $45 \mathrm{~min}$. The overall median time from prescription to administration of antibiotics was $34 \mathrm{~min}$. This is an improvement of $31 \%(15 / 49)$ from our baseline results and an improvement of $17 \%$ (7/41) from our first PDSA cycle.

The above changes helped to reduce the overall median time from GP referral to antibiotic administration to $297 \mathrm{~min}$ from a baseline of $326 \mathrm{~min}$; a reduction of $8.9 \%(29 / 326)$.

Having analysed all the notes of patients referred to AMU with possible sepsis based on their documented diagnosis on transfer from AMU, it is probable that our data are relatively complete; we may, however, have missed some patients whose diagnosis of sepsis on AMU was not recorded, but this is unlikely.

During our project, there was an international shortage of piperacillin-tazobactam, which is a common choice of broad-spectrum antibiotic in sepsis of unknown source. This shortage will make choice of antibiotics harder and may have prolonged the time from admission to antibiotic delivery. However, as we have looked at time from prescription to administration, this will not have become apparent in our results.

\section{LESSONS AND LIMITATIONS}

Having done our initial audit against NICE sepsis guidelines, we continued to use this for our inclusion criteria. In retrospect, this was an overly complicated way of identifying patients. This in part reflects the complexity of NICE guideline, which we feel will struggle to be clinically applicable.

Our sample size was much smaller than anticipated; in particular during datasets 2 and 3. Our initial data were over 2 months and during the autumn with a reasonable sample size; however, subsequent runs were over 1 month during the spring/summer with much lower patient numbers. This small sample size increases the possibility that our improvements are due to chance. This limited the study and could be overcome in future by doing runs over a longer time period or aiming for a minimum number of patients.

It would have been preferable to have done more data points; however, time delays in the implementation of our first intervention made this challenging. However, this significant system-wide implementation was essential to the sustainability of the project.

Our subsequent intervention, such as education, were cohort specific and we acknowledge these may not have sustainability. However, we hope that by being taken forwards by consultants and incorporated into departmental induction will provide some sustainability.

The rota system in use at the time of this project meant that junior doctors spent a short time rotating through AMU. As such, the junior medical staff may not have felt a part of the team and may not be aware of systems in place. This rota system has subsequently changed, which will hopefully result in a greater sense of being part of the acute medical team and hopefully improve engagement from junior medical staff in changes.

Having worked on many different AMUs, we feel that the problem we have identified and the solution we have implemented are relevant to other units.

\section{CONCLUSION}

In conclusion, our quality improvement project with small patient numbers identified a significant problem in antibiotic prescribing relevant to many AMUs. Through liaison with IT teams and pharmacy teams, we were able to implement a significant system change, with subsequent patient benefit, resulting in a sustained decrease in time from antibiotic prescription to administration of $31 \%$ and an overall reduction in time from GP referral to antibiotic administration of $8.9 \%$. By implementing a system change, we have ensured our project is sustainable and our continued evaluation of the electronic prescribing system and programme of education surrounding sepsis will further contribute to the sustainability of this project. In addition, the lessons learned on the medical admission unit can be used to improve sepsis management across the trust and within other specialties.

With increasing numbers of hospitals using electronic prescribing and record keeping, there are many opportunities to improve these systems. Many of these are created and maintained by staff that do not use the systems on a daily basis, and as such, junior doctors are the perfect people to identify problems within these systems and propose solutions that will work for the end users-the doctors themselves.

Acknowledgements The authors would like to thank Dr Alan Anthony (Consultant Acute Physician) and the team on the Acute Medical Unit and Darlington Memorial Hospital for their help and support with this project.

Contributors Both authors had equal involvement in the project design, the data collection, analysis and production of the final report.

Funding The authors have not declared a specific grant for this research from any funding agency in the public, commercial or not-for-profit sectors. 
Competing interests None declared.

Patient consent for publication Not required.

Ethics approval We did not seek ethical approval for this project as it was deemed a quality improvement project rather than a research study. This project was registered with the trust's audit office, and a report submitted for their records.

Provenance and peer review Not commissioned; externally peer reviewed.

Open access This is an open access article distributed in accordance with the Creative Commons Attribution Non Commercial (CC BY-NC 4.0) license, which permits others to distribute, remix, adapt, build upon this work non-commercially, and license their derivative works on different terms, provided the original work is properly cited, appropriate credit is given, any changes made indicated, and the use is non-commercial. See: http://creativecommons.org/licenses/by-nc/4.0/.

\section{REFERENCES}

1. Farre A, Bem D, Heath G, et al. Perceptions and experiences of the implementation, management, use and optimisation of electronic prescribing systems in hospital settings: protocol for a systematic review of qualitative studies. BMJ Open 2016;6:e011858.

2. Rhodes A, Evans LE, Alhazzani W, et al. Surviving Sepsis Campaign: international guidelines for the management of sepsis and septic shock. Crit Care Med 2016;45:486-552.

3. NCEPOD. Just say sepsis! A review of the process of care received by patients with sepsis. London: National Confidential Enquiry into Patient Outcome and Death, 2015. www.ncepod.org. uk/2015report2/ downloads/JustSaySepsis_FullReport.pdf.

4. Prescott HC, Osterholzer JJ, Langa KM, et al. Late mortality after sepsis: propensity matched cohort study. BMJ 2016;353:i2375.

5. Singer M, Deutschman CS, Seymour CW, et al. The third international consensus definitions for sepsis and septic shock (Sepsis-3). JAMA 2016;315:801-10.

6. Johnston ANB, Park J, Doi SA, et al. Effect of immediate administration of antibiotics in patients with sepsis in tertiary care: a systematic review and meta-analysis. Clin Ther 2017;39:190-202.

7. Rosenbloom ST. Approaches to evaluating electronic prescribing. $J$ Am Med Inform Assoc 2006;13:399-401.

8. NICE. Sepsis: recognition, diagnosis and early management. NG51. London: National Institute for Health and Care Excellence, 2016. https://www.nice.org.uk/guidance/ng51/resources/sepsis-reco gnition-diagnosis-and-early-management- 